\title{
Stoichiometric Network Analysis as Mathematical Method for Examinations of Instability Region and Oscillatory Dynamics
}

\author{
Ž. Čupić, G. Schmitz, Lj. Kolar-Anić
}

\begin{abstract}
Reaction systems in chemistry, physical chemistry, and biochemistry, which can be described by true or pseudo-stoichiometric relationships between species, and, therefore, represented with stoichiometric models, are usually very complex. For the analysis of the models of these complex nonlinear reaction systems with more than three variables, which can be in different dynamic states like multistability, oscillatority or chaos, some general mathematical methods such as the Stoichiometric network analysis (SNA) must be used. Although the SNA is a powerful method for systematic examination of complex reaction systems, identification of underlying reaction pathways, and stability analysis of dynamic states, this method is practically unknown among mathematicians. Therefore, a simple application of SNA to one five-dimensional model is given here.
\end{abstract}

Keywords: Stoichiometric network analysis (SNA), mathematical modeling, stoichiometric models, nonlinear oscillatory reaction system, instability condition

\section{Introduction}

In complex nonlinear reaction system it is generally possible to find one or several regions in the parameter space where the main steady state is unstable. In these regions numerous selforganized dynamic states can be observed, such as multistability, oscillatority and chaos [12-14, 22, 27-29, 31, 32, 36, 38]. Although the mentioned dissipative structures are very common in nature (for example, almost all biochemical processes are in oscillatory dynamic states $[12-15,17,22-24,26-29,31,32,36,38])$, the region of parameter space where they appear is often very narrow. Mathematical modeling of these complex processes is therefore

Manuscript received July 21, 2015; accepted February 4, 2016.

Z. Čupic is with the University of Belgrade, Institute of Chemistry, Technology and Metallurgy, Department of Catalysis and Chemical Engineering, Belgrade, Serbia; G. Schmitz is with the Faculty of Applied Sciences, University of Libre de Bruxelles, Brussels, Belgium; Lj. Kolar-Anić is with the University of Belgrade, Faculty of Physical Chemistry, Belgrade, Serbia. 
of essential importance, as it allows us to explore the parameter space systematically and identify the instability regions. If the investigated process is simple and the model can be reduced to two or three variables, the locus of unstable steady (nonequilibrium stationary) states can be easily obtained. For the analysis of models with more variables, some general methods must be used, such as the Stoichiometric Network Analysis (SNA) [3-7, 21]. The SNA is a powerful method for systematic examination of the models of complex reaction systems, identification of underlying reaction pathways, and stability analysis of dynamic states [3-12, 16-21, 23-26, 32-35]. However, this method is presented in literature in such a manner that it is not appropriately adapted to mathematicians, and, on the other side, also to chemists, physical chemist or biochemists, resulting in antagonism of scientist against this procedure [3-7]. With aim to exceed mentioned problem, we shall present here the SNA procedure applied to one five-dimensional model of a complex nonlinear reaction system that is derived from the model of the Bray-Liebhafsky oscillatory reaction $[1,2]$.

\section{Model}

Generally, in reaction systems, one or more initially present species, the reactants, give rise to one or more species that are not further transformed, the products, through a number of intermediate species that are produced and consumed during the course of the reaction. Hence, there are two fundamentally different types of species. The reactants and products are called external species. The intermediates that are produced and consumed during timeevolution of the process and do not appear in the net reaction are called internal species. The SNA studies the time evolution of the internal species for given concentrations of the external ones. In the proposed model Reactant $\mathrm{R}$ gives the product $\mathrm{P}$ via five intermediate species: $\mathrm{X}_{1}, \ldots, \mathrm{X}_{5}$.

\begin{tabular}{ll}
\hline Reactions & no. \\
\hline$X_{3} \rightarrow X_{4}+X_{5}$ & $(\mathrm{R} 1)$ \\
$X_{4}+X_{5} \rightarrow X_{3}$ & $(\mathrm{R} 2)$ \\
$X_{3}+X_{5} \rightarrow X_{2}$ & $(\mathrm{R} 3)$ \\
$X_{2} \rightarrow 2 X_{4}$ & $(\mathrm{R} 4)$ \\
$2 X_{4} \rightarrow X_{2}$ & $(\mathrm{R} 5)$ \\
$X_{3}+X_{4} \rightarrow X_{1}$ & $(\mathrm{R} 6)$ \\
$X_{1} \rightarrow X_{3}+X_{4}$ & $(\mathrm{R} 7)$ \\
$R+X_{4} \rightarrow X_{3}+P$ & $(\mathrm{R} 8)$ \\
$R+X_{2} \rightarrow X_{4}+X_{5}$ & $(\mathrm{R} 9)$ \\
\hline
\end{tabular}


Any reversible reaction can be written as two opposite forward reactions, in our case (R1) - (R2), (R4) - (R5) and (R6) - (R7). The correspondence with the chemical model [33] is given by $\mathrm{X}_{1}=\mathrm{I}_{2}, \mathrm{X}_{2}=\mathrm{I}_{2} \mathrm{O}, \mathrm{X}_{3}=\mathrm{I}^{-}, \mathrm{X}_{4}=\mathrm{HOI}$ and $\mathrm{X}_{5}=\mathrm{HIO}_{2}$.

Denoting the concentrations of the intermediates $\mathrm{X}_{i}(i=1, \ldots, 5)$ by $x_{i}$, the time evolution of the system can be described by the following set of differential equations based on the proposed model and mass-action kinetics (see Appendix 1) [30, 37]:

$$
\begin{aligned}
\frac{\mathrm{d} x_{1}}{\mathrm{~d} t} & =\mathrm{k}_{6} x_{3} x_{4}-\mathrm{k}_{7} x_{1} \\
\frac{\mathrm{d} x_{2}}{\mathrm{~d} t} & =\mathrm{k}_{3} x_{3} x_{5}-\mathrm{k}_{4} x_{2}+\mathrm{k}_{5} x_{4}^{2}-\mathrm{k}_{9} x_{2} x_{6} \\
\frac{\mathrm{d} x_{3}}{\mathrm{~d} t} & =-\mathrm{k}_{1} x_{3}+\mathrm{k}_{2} x_{4} x_{5}-\mathrm{k}_{3} x_{3} x_{5}-\mathrm{k}_{6} x_{3} x_{4}+\mathrm{k}_{7} x_{1}+\mathrm{k}_{8} x_{4} x_{6} \\
\frac{\mathrm{d} x_{4}}{\mathrm{~d} t} & =\mathrm{k}_{1} x_{3}-\mathrm{k}_{2} x_{4} x_{5}+2 \mathrm{k}_{4} x_{2}-2 \mathrm{k}_{5} x_{4}^{2}-\mathrm{k}_{6} x_{3} x_{4}+\mathrm{k}_{7} x_{1}-\mathrm{k}_{8} x_{4} x_{6}+\mathrm{k}_{9} x_{2} x_{6} \\
\frac{\mathrm{d} x_{5}}{\mathrm{~d} t} & =\mathrm{k}_{1} x_{3}-\mathrm{k}_{2} x_{4} x_{5}-\mathrm{k}_{3} x_{3} x_{5}+\mathrm{k}_{9} x_{2} x_{6}
\end{aligned}
$$

Here $\mathrm{k}_{j}(j=1, \ldots, 9)$ denote the rate constants. From a mathematical point of view, in these equations, $\mathrm{k}_{j}$ are the parameters, whereas the concentrations $x_{i}(i=1, \ldots, 5)$ of intermediate species, are variables determining dynamic state of the considered reaction system. Therefore, the number of intermediate species defines the number of differential equations necessary to describe time evolution of the examined system in the concentration phase space, and, thus, determines the dimension of the analyzed problem.

The above differential equations can be written as a function of reaction rates $v_{j}(j=$ $1, \ldots, 9)$ given by the expressions: $v_{1}=\mathrm{k}_{1} x_{3}, v_{2}=\mathrm{k}_{2} x_{4} x_{5}, v_{3}=\mathrm{k}_{3} x_{3} x_{5}, v_{4}=\mathrm{k}_{4} x_{2}, v_{5}=\mathrm{k}_{5} x_{4}^{2}$, $v_{6}=\mathrm{k}_{6} x_{3} x_{4}, v_{7}=\mathrm{k}_{7} x_{1}, v_{8}=\mathrm{k}_{8} x_{4} x_{6}$ and $v_{9}=\mathrm{k}_{9} x_{2} x_{6}$ :

$$
\begin{aligned}
\frac{\mathrm{d} x_{1}}{\mathrm{~d} t} & =v_{6}-v_{7} \\
\frac{\mathrm{d} x_{2}}{\mathrm{~d} t} & =v_{3}-v_{4}+v_{5}-v_{9} \\
\frac{\mathrm{d} x_{3}}{\mathrm{~d} t} & =-v_{1}+v_{2}-v_{3}-v_{6}+v_{7}+v_{8} \\
\frac{\mathrm{d} x_{4}}{\mathrm{~d} t} & =v_{1}-v_{2}+2 v_{4}-2 v_{5}-v_{6}+v_{7}-v_{8}+v_{9} \\
\frac{\mathrm{d} x_{5}}{\mathrm{~d} t} & =v_{1}-v_{2}-v_{3}+v_{9}
\end{aligned}
$$

Thus, we are dealing with a model given by nine stoichiometric relations between five species. The time evolution of their concentrations is described by corresponding system of 
nonlinear first order differential equations (1.1-1.5). This system of differential equations is defined by law of mass action and, therefore, it is not arbitrary. Consequently, any modifications in modelling must be performed starting from the stoichiometric model. In the mathematical literature, the system of differential equations is often taken as the model and the main postulates as well as modifications in the modelling ought to be carried out on it.

The modeling of reaction systems by stoichiometric model has important advantage, since the basic model of reaction mechanism is universal for considered process. Therefore, when modification is necessary it can be easily updated, whereas the sophistically composed system of differential equations, in the other case, must be postulated from the beginning for every new demand. The SNA is the simple method for examination of such stoichiometric models of complex nonlinear processes, identification of underlying reaction pathways, and stability analysis of dynamic states.

\section{Stoichiometric Network Analysis (SNA)}

Modeling complex nonlinear processes requires a deep understanding of dynamical system theory, beside the standard kinetic examinations. In the case of complex nonlinear reaction systems, such as oscillatory reactions, the stability of the steady states needs to be examined in different regions of the parameter space.

As the concentrations of intermediate species are the dynamical variables, their number defines the number of differential equations needed to describe the dynamics of the examined system in the concentration phase space, and, thus, determines the dimensionality of this phase space. Consequently, a stability analysis will yield a characteristic equation whose order is equal to the number $n$ of these intermediates. If a reaction system consists of two or three intermediate species $(n=2$ or 3$)$ the stability analysis can be performed simply by using the standard mathematical procedure [14] starting from the linearized kinetic equations in the vicinity of the steady states. The SNA also starts from the linearized kinetic equations in the vicinity of a steady state. However, it avoids direct solving of the $n$ dimensional characteristic equation of the Jacobian matrix which is practically unsolvable for $n>3$.

The fundamental idea of SNA is to perform the stability analysis in the rates space instead of the concentrations space. It uses new parameters derived from the analysis of reaction routes or pathways of the considered process in the steady state. Any linear combination of the elementary steps that results in some net stoichiometric reaction without intermediate species therein is one reaction route or pathway. In terminology of SNA, the steady state (or nonequilibrium stationary state) reaction pathways are currents. Clarke [35] has shown that all the currents form a convex cone in the rate space, a 9 dimensional space in our example. The edges of this cone are called extreme currents. The first aim of SNA is to determine all extreme currents. For this, we first transform kinetic equations to a 
matrix form and define the reaction pathways.

\subsection{Matrix Form of Kinetic Equations}

The differential equations (6)-(10) may be written in the matrix form (11).

$$
\begin{aligned}
& \frac{\mathrm{d} x_{1}}{\mathrm{~d} t}=0 v_{1}+0 v_{2}+0 v_{3}+0 v_{4}+0 v_{5}+1 v_{6}-1 v_{7}+0 v_{8}+0 v_{9} \\
& \frac{\mathrm{d} x_{2}}{\mathrm{~d} t}=0 v_{1}+0 v_{2}+1 v_{3}-1 v_{4}+1 v_{5}+0 v_{6}-0 v_{7}+0 v_{8}-1 v_{9} \\
& \frac{\mathrm{d} x_{3}}{\mathrm{~d} t}=-1 v_{1}+1 v_{2}-1 v_{3}+0 v_{4}+0 v_{5}-1 v_{6}+1 v_{7}+1 v_{8}+0 v_{9} \\
& \frac{\mathrm{d} x_{4}}{\mathrm{~d} t}=1 v_{1}-1 v_{2}+0 v_{3}+2 v_{4}-2 v_{5}-1 v_{6}+1 v_{7}-1 v_{8}+1 v_{9} \\
& \frac{\mathrm{d} x_{5}}{\mathrm{~d} t}=1 v_{1}-1 v_{2}-1 v_{3}+0 v_{4}+0 v_{5}+0 v_{6}+0 v_{7}+0 v_{8}+1 v_{9}
\end{aligned}
$$

The coefficients in these five equations form a matrix $\mathbf{S}$, called stoichiometric matrix.

$$
\mathbf{S}=\left[\begin{array}{rrrrrrrrr}
\mathrm{R} 1 & \mathrm{R} 2 & \mathrm{R} 3 & \mathrm{R} 4 & \mathrm{R} 5 & \mathrm{R} 6 & \mathrm{R} 7 & \mathrm{R} 8 & \mathrm{R} 9 \\
0 & 0 & 0 & 0 & 0 & 1 & -1 & 0 & 0 \\
0 & 0 & 1 & -1 & 1 & 0 & 0 & 0 & -1 \\
-1 & 1 & -1 & 0 & 0 & -1 & 1 & 1 & 0 \\
1 & -1 & 0 & 2 & -2 & -1 & 1 & -1 & 1 \\
1 & -1 & -1 & 0 & 0 & 0 & 0 & 0 & 1
\end{array}\right] \mathrm{X}_{1}
$$

Thus, the kinetic equations necessary for analysis of dynamic states of the Model (R1)(R9) have the following matrix form:

$$
\left[\begin{array}{c}
\frac{d x_{1}}{d t} \\
\frac{d x_{2}}{d t} \\
\vdots \\
\frac{d x_{5}}{d \mathrm{t}}
\end{array}\right]=S \cdot\left[\begin{array}{c}
\mathrm{v}_{1} \\
\mathrm{v}_{2} \\
\vdots \\
\mathrm{v}_{9}
\end{array}\right]
$$

The corresponding stationary equation is (14) where $\boldsymbol{v}_{s s}$ is the one-column matrix of the reaction rates at the steady state.

$$
\mathbf{S} \cdot v_{s s}=0
$$

In the general case, the concentrations of the intermediates in the steady states are functions of reactant concentrations, like $\mathrm{R}$ in our example. The SNA perform a stability analysis for given values of the reactant concentrations. In a steady state, the process as a whole 
can be presented as a linear combination of several elementary reaction pathways or reaction routes with non-negative coefficients [3,5]. These elementary reaction pathways are the extreme currents $E_{i}$. They are obtained by identification of the right null vectors of the stoichiometric matrix. In proposed model it would give:

$$
\left[\begin{array}{rrrrrrrrr}
0 & 0 & 0 & 0 & 0 & 1 & -1 & 0 & 0 \\
0 & 0 & 1 & -1 & 1 & 0 & 0 & 0 & -1 \\
-1 & 1 & -1 & 0 & 0 & -1 & 1 & 1 & 0 \\
1 & -1 & 0 & 2 & -2 & -1 & 1 & -1 & 1 \\
1 & -1 & -1 & 0 & 0 & 0 & 0 & 0 & 1
\end{array}\right] \cdot\left[\begin{array}{c}
e_{i, 1} \\
e_{i, 2} \\
\vdots \\
e_{i, 9}
\end{array}\right]=\left[\begin{array}{c}
0 \\
0 \\
\vdots \\
0
\end{array}\right]
$$

Since all elements of the vector on the right-hand side of equation (15) are equal to zero, the last expression is a homogeneous system of equations without unique solution. To avoid this problem, we use appropriate scaling relation of the reaction rates as additional equation: the sum of the $\mathrm{e}_{i}$ will be equal to one. Hence, a row with all elements equal to one is added in matrix $S$ (Eq. (4)) before calculation of extreme currents, so Eq. (15) is transformed to Eq. (16) or Eq. (17) in matrix form.

$$
\begin{aligned}
& {\left[\begin{array}{rrrrrrrrr}
0 & 0 & 0 & 0 & 0 & 1 & -1 & 0 & 0 \\
0 & 0 & 1 & -1 & 1 & 0 & 0 & 0 & -1 \\
-1 & 1 & -1 & 0 & 0 & -1 & 1 & 1 & 0 \\
1 & -1 & 0 & 2 & -2 & -1 & 1 & -1 & 1 \\
1 & -1 & -1 & 0 & 0 & 0 & 0 & 0 & 1 \\
1 & 1 & 1 & 1 & 1 & 1 & 1 & 1 & 1
\end{array}\right] \cdot\left[\begin{array}{c}
e_{i, 1} \\
e_{i, 2} \\
\vdots \\
e_{i, 9}
\end{array}\right]_{s s}=\left[\begin{array}{c}
0 \\
0 \\
0 \\
0 \\
0 \\
1
\end{array}\right]} \\
& \mathbf{B} \cdot e_{\mathrm{ss}}=\mathrm{b}
\end{aligned}
$$

Rank of the corresponding stoichiometric matrix $\mathbf{S}$ is $n=5$. The rank of matrix $B$ is equal to $n+1$. Therefore, there are $n+1$ independent columns in this matrix. Any combination of $n+1$ columns could give one independent reaction pathway in the steady state. However, some combinations lead to the same solution, others do not have solution at all and some combinations have solutions that could be decomposed to combinations of other ones. Nevertheless, all combinations of $n+1$ columns will give all extreme currents. Selection of $n+1$ from total number of $m$ columns of the matrix $S$ is equivalent to demand that other $m-(n+1) e_{i}$ are zero for the considered reaction pathway. Finally, the $e_{i}$ can be multiplied by common factor with aim to obtain small integer numbers. 
Hence, all combinations of $n+1=6$ columns of $B$ matrix will give all extreme currents. For example, first six columns have no solution whereas combination of the columns corresponding to $v_{1}, v_{3}, v_{4}, v_{6}, v_{8}$ and $v_{9}$ gives following system of equations:

$$
\left[\begin{array}{rrrrrrrrr}
0 & 0 & 0 & 0 & 0 & 1 & -1 & 0 & 0 \\
0 & 0 & 1 & -1 & 1 & 0 & 0 & 0 & -1 \\
-1 & 1 & -1 & 0 & 0 & -1 & 1 & 1 & 0 \\
1 & -1 & 0 & 2 & -2 & -1 & 1 & -1 & 1 \\
1 & -1 & -1 & 0 & 0 & 0 & 0 & 0 & 1 \\
1 & 1 & 1 & 1 & 1 & 1 & 1 & 1 & 1
\end{array}\right] \cdot\left[\begin{array}{c}
e_{i, 1} \\
0 \\
\mathrm{e}_{i, 3} \\
\mathrm{e}_{\mathrm{i}, 4} \\
0 \\
\mathrm{e}_{i, 6} \\
0 \\
\mathrm{e}_{\mathrm{i}, 8} \\
\mathrm{e}_{i, 9}
\end{array}\right]_{s s}=\left[\begin{array}{c}
0 \\
0 \\
0 \\
0 \\
0 \\
1
\end{array}\right]
$$

with solution:

$$
e_{3, s s}=e_{8, s s}=e_{9, s s}=\frac{1}{3} \quad \text { and } \quad e_{1, s s}=e_{6, s s}=0
$$

equivalent to

$$
e_{3, s s}=e_{8, s s}=e_{9, s s}=1 \quad \text { and } \quad e_{1, s s}=e_{6, s s}=0
$$

So, we have just obtained one reaction pathway in the steady state, that is, one extreme current of the stoichiometric network represented by the Model (R1)-(R9). Other combinations of columns of the matrix $B$ give three additional independent extreme currents. All mutually different irreducible solutions obtained for extreme currents are columns of the new matrix $\mathbf{E}$, where the order of columns is arbitrary: 


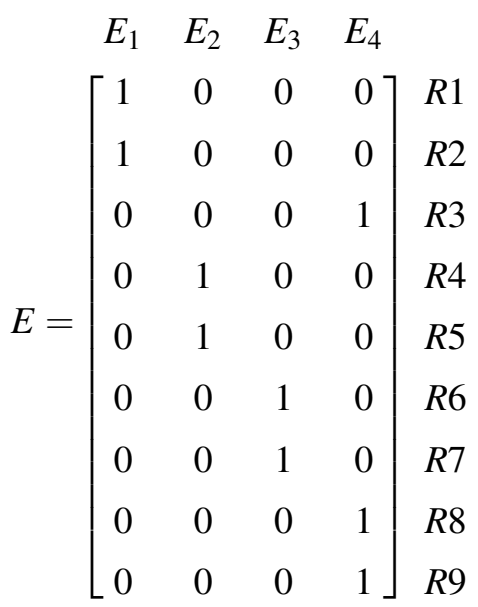

Any column of the current matrix $\mathbf{E}$ represents one combination of reaction steps (the reactions of the model) that ensure net reaction without intermediates in it. In other words, in one reaction pathway any appearance of intermediates will be exactly compensated by their disappearance. In the considered example, the reactant and product are included only in the reactions (R8) and (R9) and, therefore, only in the fourth extreme current and also in the corresponding net reaction. Summing the reactions multiplied by the elements of $E_{4}$ we get:

$$
\begin{aligned}
& X_{3}+X_{5} \rightarrow X_{2} \\
& R+X_{4} \rightarrow X_{3}+P \\
& R+X_{2} \rightarrow X_{4}+X_{5} \\
\Sigma: & 2 \mathrm{R} \longrightarrow \mathrm{P}
\end{aligned}
$$

First three extreme currents are due to three reversible reactions, (R1) and (R2), (R4) and (R5), as well as (R6) and (R7), and therefore have no stoichiometric net result. For example $E_{1}$ gives

$$
\begin{aligned}
& X_{3} \rightarrow X_{4}+X_{5} \\
& X_{4}+X_{5} \rightarrow X_{3} \\
\Sigma: & 0 \longrightarrow 0
\end{aligned}
$$

Thus, using SNA, we are able to analyze all reaction routes which is impossible to do without this method if the model is complex. 


\subsection{Current Rates}

We have just seen that any simple reaction in a stationary state can give some contribution to the reaction pathways (extreme currents) where it takes place. Therefore, any reaction rate in the stationary state $v_{\mathrm{ss}}$ can be decomposed in current rates. The contributions of the extreme currents to the reactions rates, named current rates and denoted by $j_{i}$, are the components of the corresponding vectorj. Thus, the basic equation of the stoichiometric network analysis is:

$$
v_{s s}=\mathbf{E} \cdot j
$$

Having the matrix $\mathbf{E}$, we can write the reaction rates at the steady state as linear combinations of the extreme current rates. These equations imply relations between the reaction rates at the steady state as well as the relations between the steady state concentrations. In the case of the Model (R1)-(R9) we obtain:

$$
\left[\begin{array}{l}
v_{1} \\
v_{2} \\
v_{3} \\
v_{4} \\
v_{5} \\
v_{6} \\
v_{7} \\
v_{8} \\
v_{9}
\end{array}\right]_{s s}=\left[\begin{array}{llll}
1 & 0 & 0 & 0 \\
1 & 0 & 0 & 0 \\
0 & 0 & 0 & 1 \\
0 & 1 & 0 & 0 \\
0 & 1 & 0 & 0 \\
0 & 0 & 1 & 0 \\
0 & 0 & 1 & 0 \\
0 & 0 & 0 & 1 \\
0 & 0 & 0 & 1
\end{array}\right] \cdot\left[\begin{array}{l}
j_{1} \\
j_{2} \\
j_{3} \\
j_{4}
\end{array}\right]
$$

that is:

$$
\begin{aligned}
& v_{1, s s}=v_{2, s s}=j_{1} \\
& v_{3, s s}=i t v_{8, s s}=v_{9, s s}=j_{4} \\
& v_{4, s s}=v_{5, s s}=j_{2} \\
& v_{6, s s}=v_{7, s s}=j_{3}
\end{aligned}
$$

Let us underline that, in the general case, reaction rates are linear combinations of current rates. Taking into account rates of reactions (R1)-(R9) based on mass action law, given under Eqs. (6)-(10) and (23), steady state reaction rates can be expressed by means of both classic kinetic (rate constants and steady state concentrations) or SNA (current rates) parameters: 


$$
\begin{aligned}
& v_{1, s s}=v_{2, s s}=k_{1} x_{3, s s}=k_{2} x_{4, s s} x_{5, s s}=j_{1} \\
& v_{3, s s}=v_{8, s s}=v_{9, s s}=k_{3} x_{3, s s} x_{5, s s}=k_{8} x_{4, s s} x_{6,0}=k_{9} x_{2, s s} x_{6,0}=j_{4} \\
& v_{4, s s}=v_{5, s s}=k_{4} x_{2, s s}=k_{5} x_{4, s s}^{2}=j_{2} \\
& v_{6, s s}=v_{7, s s}=k_{6} x_{3, s s} x_{4, s s}=k_{7} x_{1, s s}=j_{3}
\end{aligned}
$$

Here there are five steady state concentrations of intermediary species $\mathrm{X}_{i}(i=1, \ldots, 5)$ and the concentration of reactant $\mathrm{X}_{6}$. Since the concentration of reactant $\mathrm{X}_{6}$ is much higher than the concentration of other species, and therefore $x_{6}$ changes much more slowly than the concentration of the intermediate species, it is taken as constant in SNA with value $x_{6,0}$ in further calculations. The relations (24) are particularly useful for evaluation of the steady state concentrations. Thus, from five relations between five stationary state concentrations given in equations (24), we can easily obtain their values. They are

$$
\begin{aligned}
& x_{1, s s}=\frac{\mathrm{k}_{6}}{\mathrm{k}_{7}} \frac{\mathrm{k}_{2}}{\mathrm{k}_{1}}\left(\frac{\mathrm{k}_{8} \mathrm{k}_{4}}{\mathrm{k}_{9} \mathrm{k}_{5}}\right)^{2} \sqrt{\frac{\mathrm{k}_{8} \mathrm{k}_{1}}{\mathrm{k}_{3} \mathrm{k}_{2}} x_{6,0}} \\
& x_{2, s s}=\frac{\mathrm{k}_{5}}{\mathrm{k}_{4}}\left(\frac{\mathrm{k}_{8} \mathrm{k}_{4}}{\mathrm{k}_{9} \mathrm{k}_{5}}\right)^{2} \\
& x_{3, s s}=\frac{\mathrm{k}_{2}}{\mathrm{k}_{1}} \frac{\mathrm{k}_{8} \mathrm{k}_{4}}{\mathrm{k}_{9} \mathrm{k}_{5}} \sqrt{\frac{\mathrm{k}_{8} \mathrm{k}_{1}}{\mathrm{k}_{3} \mathrm{k}_{2}} x_{6,0}}=\frac{\mathrm{k}_{8} \mathrm{k}_{4}}{\mathrm{k}_{9} \mathrm{k}_{5}} \\
& x_{5, s s}=\sqrt{\frac{\mathrm{k}_{8} \mathrm{k}_{1}}{\mathrm{k}_{3} \mathrm{k}_{2}} x_{6,0}}
\end{aligned}
$$

The advantage of this procedure where we calculate steady state concentrations using the relations between reaction rates and current rates can be seen better on larger models, where traditional calculations are practically impossible.

\subsection{The Stability Analysis}

The next step in SNA is to investigate the stability of the network's motion around a steady state after an infinitely small perturbation, that is, to find the instability condition and the region of parameters where the steady state of the model is unstable. The dynamics of small concentration perturbations $\Delta x=\mathrm{x}-\mathrm{x}_{\mathrm{ss}}$ near a steady state $x_{\mathrm{ss}}$ is given by the equation

$$
\frac{\mathrm{d} \Delta x}{\mathrm{~d} t}=\mathbf{M} \Delta \mathbf{x}
$$


obtained by linearization of the general equation of motion about this steady state. Its stability depends on the sign of the real part of the eigenvalues of the Jacobian matrix $\mathbf{M}$ given in the form

$$
\mathbf{M}=\mathrm{S}\left(\operatorname{diag} v_{\mathrm{ss}}\right) \mathrm{K}^{\mathrm{T}}\left(\operatorname{diag} \mathbf{x}_{\mathrm{ss}}^{-1}\right)
$$

Here, $\mathbf{K}$ is the matrix of the orders of reaction and $\mathbf{K}^{T}$ is its transpose. In the SNA [3] the matrix $\mathbf{M}$ is written as

$$
\mathbf{M}=\mathrm{S}(\operatorname{diag} \mathrm{E} j) \mathbf{K}^{\mathrm{T}}(\operatorname{diag} \mathbf{h}),
$$

where $\operatorname{diag} \boldsymbol{h}$ is a diagonal matrix whose elements are the reciprocals of steady state concentrations $\left(h_{i}=1 / x_{i, s s}\right)$ and diag $\mathbf{E} j$ is a diagonal matrix whose elements are the reaction rates at the steady state. The matrix $\mathbf{M}$ written as function of the SNA parameters has particular advantages for the stability analysis since the parameters $j_{i}$ and $h_{i}$ are non-negative, what is an essential feature of the SNA. The steady state stability is determined by the eigenvalues of $\mathbf{M}$, which are the roots $\lambda$ of the characteristic polynomial

$$
\operatorname{Det}[\lambda \mathbf{I}-\mathbf{M}]=\lambda^{5}+\lambda^{4} \alpha_{1}+\lambda^{3} \alpha_{2}+\lambda^{2} \alpha_{3}+\lambda \alpha_{4}+\alpha_{5}=0
$$

where $\alpha_{i}(i=1,2, \ldots, 5)$ is the coefficient of the corresponding eigenvalue $\lambda^{5-i}$, and $\mathbf{I}$ is the unit matrix. By convention, $\alpha_{0}=1$. Since diag $\boldsymbol{h}$ introduces only scaling factors, it is useful to define a current rate matrix $\mathbf{V}(j)$, given by the expression:

$$
\mathbf{V}(j)=-S \operatorname{diag}(E j) K^{\mathrm{T}}
$$

so that $\mathbf{M}=-\mathbf{V}(j)$ (diag h). As any $\alpha_{i}$ is the sum of minors of $\boldsymbol{V}(\boldsymbol{j})$ with dimension $i$ multiplied by the product of the corresponding $h_{i}$ values, we look for a negative diagonal minors of $\mathbf{V}(j)$. If we find negative minors in one $\alpha_{i}$ we ask if the sum of negative terms can be larger than the sum of positive terms in same $\alpha_{i}$. In the model considered here, several minors can be negative so that the steady state can be unstable.

If the underlying kinetics occurs according to the law of mass action, $\mathbf{K}^{T}$ in the last equation represents the transpose of the matrix of reaction orders, whose elements are general stoichiometric coefficients of species $\mathrm{X}_{i}$ standing on the left side of the reaction $j, s_{i, j}^{\mathrm{L}}$ (see Eq. (A1.1), Appendix 1). In the considered case, the matrix $\mathbf{K}$ is 


$$
\mathbf{K}=\left[\begin{array}{lllllllll}
0 & 0 & 0 & 0 & 0 & 0 & 1 & 0 & 0 \\
0 & 0 & 0 & 1 & 0 & 0 & 0 & 0 & 1 \\
1 & 0 & 1 & 0 & 0 & 1 & 0 & 0 & 0 \\
0 & 1 & 0 & 0 & 2 & 1 & 0 & 1 & 0 \\
0 & 1 & 1 & 0 & 0 & 0 & 0 & 0 & 0
\end{array}\right]
$$

By substituting matrixes $\mathbf{K}, \mathbf{S}$, and $\mathbf{E}$ in equation (30), the matrix of currents rates $\mathbf{V}(j)$ can be derived:

$$
\mathbf{V}(j)=\left[\begin{array}{rrrrr}
j_{3} & 0 & -j_{3} & -j_{3} & 0 \\
0 & j_{2}+j_{4} & -j_{4} & -2 j_{2} & -j_{4} \\
-j_{3} & 0 & j_{1}+j_{3}+j_{4} & -j_{1}+j_{3}-j_{4} & -j_{1}+j_{4} \\
-j_{3} & -2 j_{2}-j_{4} & -j_{1}+j_{3} & j_{1}+4 j_{2}+j_{3}+j_{4} & j_{1} \\
0 & -j_{4} & -j_{1}+j_{4} & j_{1} & j_{1}+j_{4}
\end{array}\right]
$$

Steady state is unstable if and only if one root of the characteristic equation Eq. (29) has a positive real part and the general instability condition is given by the Routh-Hurwitz criteria. However, even with the simplifications of the SNA equations, these criteria are unusable for large models. In practice, it is found that we can use a much simpler criterion: we look for conditions where a coefficient $\mathrm{a}_{i}$ can be negative (a approximation) [6,21]. Moreover, the parameters $j_{i}$ and $h_{i}$ are non-negative so that any negative term is preceded by a minus sign that can be easily located in complicated equations using symbolic calculations.

Coefficients $\alpha_{i}$ of the characteristic equation Eq. (29) represent the sum of all diagonal minors $\mathrm{M}$ of dimensions $i \times i,(i=1, \ldots, 5)$ of the matrix $\mathbf{V}(j)$ multiplied by corresponding sets of reciprocal concentrations $h_{i}[12,18,20]$. Thus, coefficients $\alpha_{1}, \alpha_{2}$ and $\alpha_{5}$ are:

$$
\alpha_{1}=j_{3} h_{1}+\left(j_{2}+j_{4}\right) h_{2}+\left(j_{1}+j_{3}+j_{4}\right) h_{3}+\left(j_{1}+4 j_{2}+j_{3}+j_{4}\right) h_{4}+\left(j_{1}+j_{4}\right) h_{5}=\operatorname{Tr}(\mathbf{M})
$$




$$
\begin{aligned}
& \alpha_{2}=j_{3}\left(j_{2}+j_{4}\right) h_{1} h_{2}+\left[j_{3}\left(j_{1}+j_{3}+j_{4}\right)-j_{3}^{2}\right] h_{1} h_{3}+\left[j_{3}\left(j_{1}+4 j_{2}+j_{3}+j_{4}\right)-j_{3}^{2}\right] h_{1} h_{4}+ \\
& \quad+j_{3}\left(j_{1}+j_{4}\right) h_{1} h_{5}+\left(j_{2}+j_{4}\right)\left(j_{1}+j_{3}+j_{4}\right) h_{2} h_{3}+ \\
& +\left[\left(j_{2}+j_{4}\right)\left(j_{1}+4 j_{2}+j_{3}+j_{4}\right)-2 j_{2}\left(2 j_{2}+j_{4}\right)\right] h_{2} h_{4}+ \\
& +\left[\left(j_{2}+j_{4}\right)\left(j_{1}+j_{4}\right)-j_{4}^{2}\right] h_{2} h_{5}+ \\
& +\left[\left(j_{1}+j_{3}+j_{4}\right)\left(j_{1}+4 j_{2}+j_{3}+j_{4}\right)-\left(-j_{1}+j_{3}-j_{4}\right)\left(-j_{1}+j_{3}\right)\right] h_{3} h_{4}+ \\
& +\left[\left(j_{1}+j_{3}+j_{4}\right)\left(j_{1}+j_{4}\right)-\left(-j_{1}+j_{4}\right)^{2}\right] h_{3} h_{5}+ \\
& +\left[\left(j_{1}+4 j_{2}+j_{3}+j_{4}\right)\left(j_{1}+j_{4}\right)-j_{1}^{2}\right] h_{4} h_{5}= \\
& =j_{3}\left(j_{2}+j_{4}\right) h_{1} h_{2}+j_{3}\left(j_{1}+j_{4}\right) h_{1} h_{3}+j_{3}\left(j_{1}+4 j_{2}+j_{4}\right) h_{1} h_{4}+ \\
& +j_{3}\left(j_{1}+j_{4}\right) h_{1} h_{5}+\left(j_{2}+j_{4}\right)\left(j_{1}+j_{3}+j_{4}\right) h_{2} h_{3}+ \\
& +\left[j_{2}\left(j_{1}+j_{3}+3 j_{4}\right)+j_{4}\left(j_{1}+j_{3}+j_{4}\right)\right] h_{2} h_{4}+ \\
& +\left[\left(j_{2}+j_{4}\right) j_{1}+j_{2} j_{4}\right] h_{2} h_{5}+\left[4\left(j_{1} j_{2}+j_{1} j_{3}+j_{2} j_{3}\right)+j_{4}\left(j_{1}+4 j_{2}+3 j_{3}+j_{4}\right)\right] h_{3} h_{4}+ \\
& +\left[4 j_{1} j_{4}+j_{3}\left(j_{1}+j_{4}\right)\right] h_{3} h_{5}+\left[j_{1}\left(4 j_{2}+j_{3}+j_{4}\right)+j_{4}\left(j_{1}+4 j_{2}+j_{3}+j_{4}\right)\right] h_{4} h_{5} \\
& \quad \alpha_{5}=2 j_{1} j_{2} j_{3} j_{4}^{2} h_{1} h_{2} h_{3} h_{4} h_{5}
\end{aligned}
$$

Obviously, the coefficients $\alpha_{1}, \alpha_{2}$ and $\alpha_{5}$ are always positive and therefore the source of instability is not in them. However, $\alpha_{3}$ and $\alpha_{4}$ may be negative. Instead to calculate all $\alpha_{i}$, we shall look for negative minors in matrix $\mathbf{V}(j)$. There are three possibly negative minors:

$$
\begin{aligned}
M_{245} & =2 j_{1} j_{4}^{2}-j_{2} j_{4}^{2}+j_{1} j_{2} j_{3}+8 j_{1} j_{2} j_{4}+j_{1} j_{3} j_{4}+j_{2} j_{3} j_{4} . \\
M_{1245} & =2 j_{1} j_{3} j_{4}^{2}-j_{2} j_{3} j_{4}^{2}+8 j_{1} j_{2} j_{3} j_{4} . \\
M_{2345} & =2 j_{1} j_{2} j_{4}^{2}-2 j_{2} j_{3} j_{4}^{2}+8 j_{1} j_{3} j_{4}^{2}+23 j_{1} j_{2} j_{3} j_{4} .
\end{aligned}
$$

Thus, only $\alpha_{3}$ and $\alpha_{4}$ can be negative. When negative term is dominant, corresponding $\alpha_{i}$ is negative, and there exists positive real part of some root of the characteristic equation. Under these conditions, the examined steady state is unstable. Since minors $\mathbf{M}_{1245}$ and $\mathbf{M}_{2345}$, both include minor $\mathbf{M}_{245}$, the source of instability is in the last one. The instability condition which results from minor $\mathrm{M}_{245}$ is:

$$
j_{2} j_{4}^{2}>2 j_{1} j_{4}^{2}+j_{1} j_{2} j_{3}+8 j_{1} j_{2} j_{4}+j_{1} j_{3} j_{4}+j_{2} j_{3} j_{4} .
$$

Besides, term $\mathrm{M}_{245} h_{2} h_{4} h_{5}$ must be dominant if we want to have negative $\alpha_{3}$. Since, $h_{i}$ being reciprocal concentrations, this term is dominant if the concentrations of the intermediate species $\mathrm{X}_{2}, \mathrm{X}_{4}$ and $\mathrm{X}_{5}$ are sufficiently low. This is true for the considered chemical system so that the instability is approximately defined by the simplified instability condition (39). The minor $\mathrm{M}_{245}$ is the core of the instability of the model. 
By means of relations (23), the instability condition (39) can be rewritten in function of the steady state reaction rates. There are several combinations of the reaction rates that satisfy mentioned equations. We can select any of them, but we always prefer the one for which we have the best experimental evidence. One possible expression for the instability condition is:

$$
v_{5} v_{3}^{2}>2 v_{1} v_{3}^{2}+v_{1} v_{5} v_{7}+8 v_{1} v_{5} v_{3}+v_{1} v_{7} v_{3}+v_{5} v_{7} v_{3}
$$

The subscript "ss" is dropped in the last expression for simplicity. The equation (40) can be also written in dimensionless form. Such kind of discussion is important for practical purposes and, although it is not necessary for further calculations here, the dimensionless kinetic equations are given in Appendix 2.

Thus, since any polynomial of related minor can be expressed as function of steady state reaction rates, by SNA we can evaluate the instability condition as function of them and correlate it with experimentally obtained values for corresponding rate constants and steady state concentrations. Moreover, as the steady state concentrations are also functions of rate constants, we can express the instability condition as function of rate constants and initial concentrations of reactants, only. Calculated instability is located in a defined domain of the phase space where we can analyze dynamic states by numerical simulations. Without mentioned calculations we had a serious problem to find the instability region, since the parameters, rate constants, differs from one another for several (frequently more than ten) order of magnitudes.

Moreover, the SNA allows the distinction between different kinds of instability. Taking into account the above calculated $\alpha_{i}$ we can construct all Hurwitz determinants $?_{i}, i=$ $1, \ldots, n$, which are defined as the determinants of the leading principal minor, made from the first $i$ rows and columns of the Hurwitz matrix $\mathbf{H}$, where $a_{i}=0$ for $i>n$ (in the analyzed model $n=5$ ).

$$
\mathbf{H}=\left[\begin{array}{cccccc}
\alpha_{1} & \alpha_{3} & \alpha_{5} & \alpha_{7} & \ldots & \alpha_{2 n-1} \\
1 & \alpha_{2} & \alpha_{4} & \alpha_{6} & \ldots & \alpha_{2 n-2} \\
0 & \alpha_{1} & \alpha_{3} & \alpha_{5} & \ldots & \alpha_{2 n-3} \\
0 & 1 & \alpha_{2} & \alpha_{4} & \ldots & \alpha_{2 n-4} \\
0 & 0 & \alpha_{1} & \alpha_{3} & \ldots & \alpha_{2 n-5} \\
\vdots & \vdots & \vdots & \vdots & \ddots & \vdots \\
0 & 0 & 0 & 0 & \ldots & \alpha_{n}
\end{array}\right]
$$

A saddle-node bifurcation occurs when the condition $\alpha_{\mathrm{n}}=0$ is satisfied [24]. For the considered model, 


$$
\alpha_{n}=\alpha_{5}=2 j_{1} j_{2} j_{3} j_{4}^{2} h_{1} h_{2} h_{3} h_{4} h_{5}>0
$$

Thus, a saddle-node bifurcation doesn't occur. An Andronov-Hopf bifurcation occurs when $\Delta_{\mathrm{n}-1}=0[7,24]$. For the considered model,

$$
\Delta_{4}=\operatorname{det}\left[\begin{array}{cccc}
\alpha_{1} & \alpha_{3} & \alpha_{5} & 0 \\
1 & \alpha_{2} & \alpha_{4} & 0 \\
0 & \alpha_{1} & \alpha_{3} & \alpha_{5} \\
0 & 1 & \alpha_{2} & \alpha_{4}
\end{array}\right]=0
$$

or

$$
\Delta_{4}=\left(\alpha_{1} \alpha_{2} \alpha_{3} \alpha_{4}+2 \alpha_{1} \alpha_{4} \alpha_{5}+\alpha_{2} \alpha_{3} \alpha_{5}\right)-\left(\alpha_{1} \alpha_{2}^{2} \alpha_{5}+\alpha_{1}^{2} \alpha_{4}^{2}+\alpha_{3}^{2} \alpha_{4}+\alpha_{5}^{2}\right)=0
$$

Both, $\alpha_{3}$ and $\alpha_{4}$ can be negative, probably simultaneously, while the other coefficients are always positive. Grouping the positive terms on the left and the potentially negative ones on the right side of equation, the condition for the Andronov-Hopf bifurcation becomes:

$$
\alpha_{1} \alpha_{2} \alpha_{3} \alpha_{4}-\alpha_{3}^{2} \alpha_{4}=\left(\alpha_{1} \alpha_{2}^{2} \alpha_{5}+\alpha_{1}^{2} \alpha_{4}^{2}+\alpha_{5}^{2}\right)-\left(2 \alpha_{1} \alpha_{4} \alpha_{5}+\alpha_{2} \alpha_{3} \alpha_{5}\right)
$$

Minor $\mathrm{M}_{245}$ remains the most probable source of the instability, and hence, the instability condition (39) or (40) can be used for the approximate identification of the AndronovHopf bifurcation.

\section{Conclusion}

The stoichiometric network analysis is applied to a five-dimensional model of a complex nonlinear reaction system with aim to attract readers to use this method in any other mathematical modeling of reaction systems that can be presented by stoichiometric relations between species. In particular, the analytical procedure for calculations of reaction pathways, instability condition and dimensionless equations, all in a model with five variables, was explicitly shown. Practical SNA-based approach is also given, to differentiate between saddle node and Andronov-Hopf bifurcations leading to instability and oscillations.

\section{Appendix 1: Differential equations based on the mass-action kinetics, matrix form of kinetic equations and stationary states}

Any reaction system consisted of $m$ reactions and $n_{\text {tot }}$ species denoted by $X_{i}\left(i=1, \ldots, n_{\text {tot }}\right)$, where $n_{\text {tot }}$ is the total number of species including both internal $(n)$ and external $\left(n_{\text {ex }}\right)$ ones, can be presented in the following form: 


$$
\begin{aligned}
& s_{1,1}^{L} X_{1}+s_{2,1}^{L} X_{2}+\cdots+s_{n_{t o t}, 1}^{L} X_{n_{\text {tot }}} \rightarrow s_{1,1}^{D} X_{1}+s_{2,1}^{D} X_{2}+\cdots+s_{n_{t o t}, 1}^{D} X_{n_{\text {tot }}} \\
& s_{1,2}^{L} X_{1}+s_{2,2}^{L} X_{2}+\cdots+s_{n_{t o t}, 2}^{L} X_{n_{t o t}} \rightarrow s_{1,2}^{D} X_{1}+s_{2,2}^{D} X_{2}+\cdots+s_{n_{t o t}, 2}^{D} X_{n_{t o t}} \\
& s_{1, m}^{L} X_{1}+s_{2, m}^{L} X_{2}+\cdots+s_{n_{t o t}, m}^{L} X_{n_{t o t}} \rightarrow s_{1, m}^{D} X_{1}+s_{2, m}^{D} X_{2}+\cdots+s_{n_{t o t}, m}^{D} X_{n_{t o t}}
\end{aligned}
$$

Here, $s_{i, j}^{\mathrm{L}}$ and $s_{i, j}^{\mathrm{D}}$ are the stoichiometric coefficients of considered species at left (L) and right (D) side of equation. Index $j$ denotes the ordinal number of a reaction in a series of $m$ reactions. The stoichiometric coefficients are usually small integer numbers $\left(s_{i, j}=\right.$ $0,1,2, \ldots)$. If stoichiometric coefficient is zero at one or both sides of equation, this species does not appear in stoichiometric expression at this or both sides, respectively. Besides, one species can appear on both sides of chemical reaction, as it is the case in direct autocatalytic or autoinhibitory reactions. All reactions can be either simple forward or reversible ones. Any reversible reaction can be written as two forward ones. In SNA, the reverse reactions must be taken as two forward ones. Therefore we shall consider the system of forward reactions only.

Denoting by $x_{i}$ the concentration of species $\mathrm{X}_{i}$, and by $\mathrm{S}_{i, j}=s_{i, j}^{\mathrm{D}}-s_{i, j}^{\mathrm{L}}$ the difference between its stoichiometric coefficients from the right and left side of reaction $j$, we can write the kinetic equations that correspond to the model (A1.1) in the following form:

$$
\begin{aligned}
& \frac{d x_{1}}{d t}=S_{1,1} v_{1}+S_{1,2} v_{2}+\cdots+S_{1, m} v_{m} \\
& \frac{d x_{2}}{d t}=S_{2,1} v_{1}+S_{2,2} v_{2}+\cdots+S_{2, m} v_{m} \\
& \quad \vdots \\
& \frac{d x_{n_{\mathrm{tot}}}}{d t}=S_{n_{\mathrm{tot}}, 1} v_{1}+S_{n_{\mathrm{tot}}, 2} v_{2}+\cdots+S_{n_{\mathrm{tot}}, m} v_{m}
\end{aligned}
$$

Here the reaction rates $v_{j}$ are functions of rate constants $\mathrm{k}_{j}$ and concentrations of the species that take place in reaction step $j$. They are usually expressed as the product of the corresponding rate constant and relevant species concentrations with respective exponents equal to stoichiometric coefficient:

$$
v_{j}=k_{j} x_{1}^{s_{1, j}^{L}} x_{2}^{s_{2, j}^{L}} \cdots x_{n_{t o t}}^{s_{n_{t o t}, j}}
$$

Let us consider reaction rates of three reactions:

\begin{tabular}{lll}
\hline Reactions & & no. \\
\hline$X_{2} \rightarrow 2 X_{4}$ & $v_{4}=\mathrm{k}_{4} x_{2}$ & $(\mathrm{R} 4)$ \\
$2 X_{4} \rightarrow X_{2}$ & $v_{5}=\mathrm{k}_{5} x_{4}^{2}$ & $(\mathrm{R} 5)$ \\
$X_{2}+2 X_{4} \rightarrow 3 X_{4}$ & $v_{10}=\mathrm{k}_{10} x_{2} x_{4}^{2}$ & $(\mathrm{R} 10)$ \\
\hline
\end{tabular}

If we imagine above three reactions as the model of a process, the time evolution of the particular species $\mathrm{X}_{2}$ and $\mathrm{X}_{4}$ is described by the following differential equations: 


$$
\begin{aligned}
& \frac{d x_{2}}{d t}=-k_{4} x_{2}+k_{5} x_{4}^{2}-k_{10} x_{2} x_{4}^{2} \\
& \frac{d x_{4}}{d t}=2 k_{4} x_{2}-2 k_{5} x_{4}^{2}+k_{10} x_{2} x_{4}^{2}
\end{aligned}
$$

The sign + in the second equation before the rate of reaction (R10) is a consequence of the fact that in this reaction there are three molecules of species $\mathrm{X}_{4}$ on the right side and two of them on the left side. That is, the concentration of this species in considered reaction will increase.

In the general case, the kinetic Eq. (A1.2) can be simply written in the matrix form, by the operator named the stoichiometric matrix $S_{\text {tot }}$ :

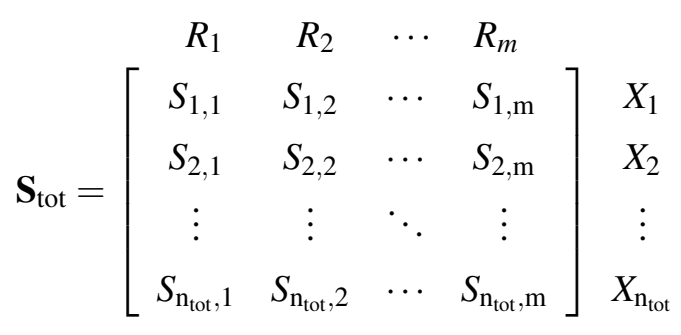

In the literature, the elements $\mathrm{S}_{i, j}=s_{i, j}^{\mathrm{D}}-s_{i, j}^{\mathrm{L}}$. are usually called stoichiometric coefficients, although there is obvious difference between $S_{i, j}$ and stoichiometric coefficients $s_{i, j}^{\mathrm{D}}$ and $s_{i, j}^{\mathrm{L}}$.

In Eq. (A1.5), the row over the matrix $S_{\text {tot }}$ denotes reactions, whereas the column on the right side denotes reaction species. This extra row and column are not part of the matrix $S_{\text {tot }}$, but help a reader to correlate their elements with corresponding reactions and species, selected in arbitrary order.

Since, in stoichiometric network analysis, the reverse reactions must be taken as two forward ones, every reverse reaction has two columns in stoichiometric matrix $S_{\text {tot }}$. The system of kinetic equations in the matrix form is:

$$
\left[\begin{array}{c}
\frac{d x_{1}}{d t} \\
\frac{d x_{2}}{d t} \\
\vdots \\
\frac{d x_{n_{\mathrm{tot}}}}{d t}
\end{array}\right]=\left[\begin{array}{cccc}
S_{1,1} & S_{1,2} & \cdots & S_{1, m} \\
S_{2,1} & S_{2,2} & \cdots & S_{2, m} \\
\vdots & \vdots & \ddots & \vdots \\
S_{n_{\mathrm{tot}}, 1} & S_{n_{\mathrm{tot}}, 2} & \cdots & S_{n_{\mathrm{tot}}, m}
\end{array}\right] \cdot\left[\begin{array}{c}
v_{1} \\
v_{2} \\
\vdots \\
v_{m}
\end{array}\right]
$$

The same equation can also be written in the form:

$$
\frac{\mathrm{d} x}{\mathrm{~d} t}=\mathbf{S}_{\mathrm{tot}} \cdot v
$$

where $\mathrm{d} x / \mathrm{d} t$ is vector, that is, one-column reaction rate matrix.

In the equilibrium stationary state, or, simply, in the equilibrium, for any reaction species $\mathrm{X}_{i}$, including reactants and products, the following equation must be satisfied:

$$
\left(\frac{d x_{i}}{d t}\right)_{s s}=S_{i, 1} v_{1, s s}+S_{i, 2} v_{2, s s}+\cdots+S_{i, m} v_{m, s s}=0
$$


Consequently, the equilibrium stationary state can be calculated from the following matrix relation:

$$
S_{t o t} \cdot v_{s s}=0
$$

In the nonequilibrium stationary state, same equation must be satisfied, but only for independent intermediates $\mathrm{X}_{i}$. If $\left(d x_{i} / d t\right)_{s s}$ for all of them are equal to zero, we shall obtain the main steady state. However, we can discuss also the steady states with respect to subsystems of independent intermediate species.

Thus, for the system of $n$ independent intermediate species, the nonequilibrium stationary state is defined by the following matrix equation:

$$
\left[\begin{array}{c}
\frac{d x_{1}}{d t} \\
\frac{d x_{2}}{d t} \\
\vdots \\
\frac{d x_{n}}{d t}
\end{array}\right]_{s s}=\left[\begin{array}{cccc}
S_{1,1} & S_{1,2} & \cdots & S_{1, m} \\
S_{2,1} & S_{2,2} & \cdots & S_{2, m} \\
\vdots & \vdots & \ddots & \vdots \\
S_{n, 1} & S_{n, 2} & \cdots & S_{n, m}
\end{array}\right] \cdot\left[\begin{array}{c}
v_{1} \\
v_{2} \\
\vdots \\
v_{m}
\end{array}\right]_{s s}=\left[\begin{array}{c}
0 \\
0 \\
\vdots \\
0
\end{array}\right]
$$

or in the following matrix form:

$$
S \cdot v_{s s}=0
$$

Thus, equation (A1.9) is related to equilibrium, while equation (A1.11) is related to the non-equilibrium stationary states, that is, the steady states. As we have already said, the equilibrium stationary states can be only stable and therefore uninteresting for further examinations of dynamic states of a system.

\section{Appendix 2: Dimensionless kinetic equations}

The dimensionless kinetic equations can be very useful. Among others, they can be used to reduce the number of parameters. The procedure for obtaining the dimensionless kinetic equations will be again demonstrated using the Model (R1)-(R9) as an example. There, we first need to define new dimensionless variables denoted for internal by $\beta_{i}$ where $\beta_{i}=x_{i} / x_{i, \mathrm{ss}}$ $(i=1, \ldots, 5)$, for reactant by $\beta_{6}=x_{6} / x_{6,0}$ and for product by $\beta_{7}=x_{7} / x_{6,0}$. Introducing them into the kinetic equations (6)-(10) we obtain:

$$
\begin{aligned}
& x_{1, s s} \frac{d \beta_{1}}{d t}=v_{6}-v_{7} \\
& x_{2, s s} \frac{d \beta_{2}}{d t}=v_{3}-v_{4}+v_{5}-v_{9} \\
& x_{3, s s} \frac{d \beta_{3}}{d t}=-v_{1}+v_{2}-v_{3}-v_{6}+v_{7}+v_{8} \\
& x_{4, s s} \frac{d \beta_{4}}{d t}=v_{1}-v_{2}+2 v_{4}-2 v_{5}-v_{6}+v_{7}-v_{8}+v_{9} \\
& x_{5, s s} \frac{\mathrm{d} \beta_{5}}{\mathrm{~d} t}=v_{1}-v_{2}-v_{3}+v_{9} \\
& x_{6,0} \frac{d \beta_{6}}{d t}=-v_{8}-v_{9} \\
& x_{6,0} \frac{d \beta_{7}}{d t}=v_{8}
\end{aligned}
$$


The reaction rates presented here can be expressed in function of dimensionless concentrations, too, and the current rates in the steady state (24):

$$
\begin{aligned}
& v_{1}=k_{1} x_{3, s s} \frac{x_{3}}{x_{3, s s}}=v_{1, s s} \beta_{3}=j_{1} \beta_{3} \\
& v_{2}=k_{2} x_{4, s s} x_{5, s s} \frac{x_{4}}{x_{4, s s}} \frac{x_{5}}{x_{5, s s}}=v_{2, s s} \beta_{4} \beta_{5}=j_{1} \beta_{4} \beta_{5} \\
& v_{3}=k_{3} x_{3, s s} x_{5, s s} \frac{x_{3}}{x_{3, s s}} \frac{x_{5}}{x_{5, s s}}=v_{3, s s} \beta_{3} \beta_{5}=j_{4} \beta_{3} \beta_{5} \\
& v_{4}=k_{4} x_{2, s s} \frac{x_{2}}{x_{2, s s}}=v_{4, s s} \beta_{2}=j_{2} \beta_{2} \\
& v_{5}=k_{5} x_{4, s s}^{2}\left(\frac{x_{4}}{x_{4, s s}}\right)^{2}=v_{5, s s} \beta_{4}^{2}=j_{2} \beta_{4}^{2} \\
& v_{6}=k_{6} x_{3, s s} x_{4, s s} \frac{x_{3}}{x_{3, s s}} \frac{x_{4}}{x_{4, s s}}=v_{6, s s} \beta_{3} \beta_{4}=j_{3} \beta_{3} \beta_{4} \\
& v_{7}=k_{7} x_{1, s s} \frac{x_{1}}{x_{1, s s}}=v_{7, s s} \beta_{1}=j_{3} \beta_{1} \\
& v_{8}=k_{8} x_{4, s s} x_{6,0} \frac{x_{4}}{x_{4, s s}} \frac{x_{6}}{x_{6,0}}=v_{8, s s} \beta_{4} \beta_{6}=j_{4} \beta_{4} \beta_{6} \\
& v_{9}=k_{9} x_{2, s s} x_{6,0} \frac{x_{2}}{x_{2, s s}} \frac{x_{6}}{x_{6,0}}=v_{9, s s} \beta_{2} \beta_{6}=j_{4} \beta_{2} \beta_{6}
\end{aligned}
$$

The obtained reaction rates in the steady state can be introduced in the kinetic equation (A2.1). Further, dividing both sides of every equation with concentrations of the species that appears on the left-hand side, we shall obtain their reciprocal concentration values on the right-hand side. As already mentioned, they are new parameters in SNA, denoted by $h_{i}$. In the case of the considered model they are:

$$
h_{i}=\frac{1}{x_{i, s}} \text {, where } i=1, \ldots, 5 \text {, and } h_{6}=\frac{1}{x_{6,0}} .
$$

Taking into account relations (A2.2) and (A2.3) the kinetic equations (A2.1) can be written as function of the SNA parameters, but they are not yet in the dimensionless form. With this aim we shall introduce the dimensionless time by means of the rate constant of any first order reaction, for example reaction (R7):

$$
d \tau=d t \cdot k_{7}=d t \cdot k_{7} x_{1, s s} \frac{1}{x_{1, s s}}=d \mathrm{t} \cdot \mathrm{v}_{7, s s} \mathrm{~h}_{1}=d \mathrm{t} \cdot \mathrm{j}_{3} \mathrm{~h}_{1} .
$$

By substitution of all relations (A2.2), (A2.3) and (A2.4) into the Eq. (A2.1), we can simply obtain the dimensionless kinetic equations in the following form:

$$
\begin{aligned}
& \frac{d \beta_{1}}{d \tau}=\beta_{3} \beta_{4}-\beta_{1} \\
& \frac{d \beta_{2}}{d \tau}=\frac{h_{2}}{h_{1}}\left(\frac{j_{4}}{j_{3}} \beta_{3} \beta_{5}-\frac{j_{2}}{j_{3}} \beta_{2}+\frac{j_{2}}{j_{3}} \beta_{4}^{2}-\frac{j_{4}}{j_{3}} \beta_{2} \beta_{6}\right) \\
& \frac{d \beta_{3}}{d \tau}=\frac{h_{3}}{h_{1}}\left(-\frac{j_{1}}{j_{3}} \beta_{3}+\frac{j_{1}}{j_{3}} \beta_{4} \beta_{5}-\frac{j_{4}}{j_{3}} \beta_{3} \beta_{5}-\beta_{3} \beta_{4}+\beta_{1}+\frac{j_{4}}{j_{3}} \beta_{4} \beta_{6}\right) \\
& \frac{d \beta_{4}}{d \tau}=\frac{h_{4}}{h_{1}}\left(\frac{j_{1}}{j_{3}} \beta_{3}-\frac{j_{1}}{j_{3}} \beta_{4} \beta_{5}+2 \frac{j_{2}}{j_{3}} \beta_{2}-2 \frac{j_{2}}{j_{3}} \beta_{4}^{2}-\beta_{3} \beta_{4}+\beta_{1}-\frac{j_{4}}{j_{3}} \beta_{4} \beta_{6}+\frac{j_{4}}{j_{3}} \beta_{2} \beta_{6}\right) \\
& \frac{d \beta_{5}}{d \tau}=\frac{h_{5}}{h_{1}}\left(\frac{j_{1}}{j_{3}} \beta_{3}-\frac{j_{1}}{j_{3}} \beta_{4} \beta_{5}-\frac{j_{4}}{j_{3}} \beta_{3} \beta_{5}+\frac{j_{4}}{j_{3}} \beta_{2} \beta_{6}\right) \\
& \frac{d \beta_{6}}{d \tau}=\frac{h_{6}}{h_{1}}\left(-\frac{j_{4}}{j_{3}} \beta_{4} \beta_{6}-\frac{j_{4}}{j_{3}} \beta_{2} \beta_{6}\right) \\
& \frac{d \beta_{7}}{d \tau}=\frac{h_{6}}{h_{1}} \frac{j_{4}}{j_{3}} \beta_{4} \beta_{6}
\end{aligned}
$$

These equations can be additionally simplified by introduction of new dimensionless 
parameters for any combination of current rates that appear several times. In the considered case they are:

$$
\kappa_{1}=\frac{j_{1}}{j_{3}}, \kappa_{2}=\frac{j_{2}}{j_{3}} \text { and } \kappa_{3}=\frac{j_{4}}{j_{3}} .
$$

Hence, the dimensionless equations can be transformed to the form:

$$
\begin{aligned}
& \frac{d \beta_{1}}{d \tau}=\beta_{3} \beta_{4}-\beta_{1} \\
& \frac{d \beta_{2}}{d \tau}=\frac{h_{2}}{h_{1}}\left(\kappa_{3} \beta_{3} \beta_{5}-\kappa_{2} \beta_{2}+\kappa_{2} \beta_{4}^{2}-\kappa_{3} \beta_{2} \beta_{6}\right) \\
& \frac{d \beta_{3}}{d \tau}=\frac{h_{3}}{h_{1}}\left(-\kappa_{1} \beta_{3}+\kappa_{1} \beta_{4} \beta_{5}-\kappa_{3} \beta_{3} \beta_{5}-\beta_{3} \beta_{4}+\beta_{1}+\kappa_{3} \beta_{4} \beta_{6}\right) \\
& \frac{d \beta_{4}}{d \tau}=\frac{h_{4}}{h_{1}}\left(\kappa_{1} \beta_{3}-\kappa_{1} \beta_{4} \beta_{5}+2 \kappa_{2} \beta_{2}-2 \kappa_{2} \beta_{4}^{2}-\beta_{3} \beta_{4}+\beta_{1}-\kappa_{3} \beta_{4} \beta_{6}+\kappa_{3} \beta_{2} \beta_{6}\right) \\
& \frac{d \beta_{5}}{d \tau}=\frac{h_{5}}{h_{1}}\left(\kappa_{1} \beta_{3}-\kappa_{1} \beta_{4} \beta_{5}-\kappa_{3} \beta_{3} \beta_{5}+\kappa_{3} \beta_{2} \beta_{6}\right) \\
& \frac{d \beta_{6}}{d \tau}=\frac{h_{6}}{h_{1}}\left(-\kappa_{3} \beta_{4} \beta_{6}-\kappa_{3} \beta_{2} \beta_{6}\right) \\
& \frac{d \beta_{7}}{d \tau}=\frac{h_{6}}{h_{1}} \kappa_{3} \beta_{4} \beta_{6}
\end{aligned}
$$

The dimensionless kinetic equations immediately show if the considered variable is slow or fast. Thus, in the considered case, concentration of the intermediate species $\mathrm{X}_{1}$ has much higher steady state value than $\mathrm{X}_{2}$, and hence, the scaling factor $h_{1}$ in the corresponding kinetic equation is much lower. For this reason species $\mathrm{X}_{1}$ will be the slow one, whereas species $\mathrm{X}_{2}$ will be the fast one.

\section{References}

[1] W. C. BRAY, A periodic reaction in homogeneous solution and its relation to catalysis, J. Am. Chem. Soc. Vol. 43 (1921) 1262-1267.

[2] W. C. BRAY, H. A.LIEBHAFSKY, Reactions involving hydrogen peroxide, iodine and iodate ion. I. Introduction,J. Am. Chem. Soc. Vol. 53 (1931) 38-44.

[3] B. L. Clarke, Stability of complex reaction networks, Advances in Chemical Physics, (I. Prigogine, S. A. Rice, eds.), John Wiley and Sons, Inc: New York, NY, Vol. 43, 1980, 1-216.

[4] B. L. Clarke, J. Clark, Chemical reaction networks with finite attractor regions, J. Chem. Phys. Vol. 82 (1985) 3107-3117.

[5] B. L. Clarke, Stoichiometric network analysis, Cell Biophys. Vol. 12 (1988) 237-253.

[6] B. CLARKE, General method for simplifying chemical networks while preserving overall stoichiometry in reduced mechanisms, J. Chem. Phys. 97 (1992) 2459-2472

[7] B. L. Clarke, W. JiAng, Method for deriving Hopf and saddle-node bifurcation hypersurfaces and application to a model of the Belousov-Zhabotinskii system, J. Chem. Phys. Vol. 99 (1993) 4464-4478.

[8] Ž. ČUPIĆ, LJ. KolaR-ANIĆ, Contraction of the model for the Bray-Liebhafsky oscillatory reaction by eliminating intermediate $I_{2} O$, J. Chem. Phys. Vol. 110 (1999) 3951-3954.

[9] Ž. ČUPIĆ, LJ. KolaR-ANIĆ, Contraction of complex models by the stoichiometric network analysis, Advanced Science and Technology of Sintering, (B. D. Stojanović, V. V. Skorokhod, M. V. Nikolić, eds), Kluwer Academic/Plenum Publishers, New York, 1999, 75-80. 
[10] Ž. ČUPIĆ, Stehiometrijska mrena analiza autokatalatora, Nauka-Tehnika-Bezbednost Vol. 2 (2001) 29-37.

[11] Ž. ČUPIĆ, Terorizam u svetlu nelinearnih nauka - Stehiometrijska mrena analiza populacionih procesa, Nauka tehnika bezbednost Vol. 2 (2003) 39-49.

[12] Ž. ČUPiĆ, V. MARKović, A. IVAnović, LJ. Kolar-AniĆ, Modeling of the Complex Nonlinear Processes: Determination of the Instability Region by the Stoichiometric Network Analysis, Mathematical Modelling, (C. R. Brennan, ed.), Nova Science Publishers, Inc. New York, 2011, 111-178.

[13] I. R. Epstein, J. A. Pojman, An Introduction to Nonlinear Chemical Dynamics. Oscillations, Waves, Patterns, and Chaos, Oxford University Press, Oxford, 1998.

[14] P. Gray, S. K. Scott, Chemical Oscillations and Instabilities, Clarendon Press, Oxford, 1990.

[15] S. Jelić, Ž. Čupić, LJ. KolaR-Anić, Mathematical modelling of the hypothalamicpituitary-adrenal system activity, Math. Biosci. Vol. 197 (2005) 173-187.

[16] S. Jelić, Ž. Čupić, Lu. KolaR-Anić, Modelling of the Hypothalamic-Pituitary-Adrenal System Activity Based on the Stoichiometric Analysis, New Research on Neurosecretory Systems, (E. Romano, S. De Luca, eds), Nova Science Publishers, Inc. New York, 2008, 225-245.

[17] S. Jelić, Ž. Čupić, Lu. Kolar-Anić, V. Vukojević, Predictive modeling of the hypothalamic-pituitary-adrenal (HPA) function. Dynamic systems theory approach by stoichiometric network analysis and quenching small amplitude oscillations, Int. J. Nonlin. Sci. Num. Simul. Vol. 10 (2009) 1451-1472.

[18] Lu. Kolar-Anić, G. Schmitz, Mechanism of the Bray-Liebhafsky reaction: Effect of the oxidation of iodous acid by hydrogen peroxide, J Chem. Soc. Faraday Trans. Vol. 88 (1992) 2343-2349.

[19] Lj. Kolar-Anić, DJ. MiŠLjenović, S. Anić, G. NicOlis, The influence of the reduction of iodate ion by hydrogen peroxide on the model of the Bray-Liebhafsky reaction, React. Kinet. Catal. Lett. Vol. 54, (1995) 35-41.

[20] Lu. Kolar-Anić, Ž. Čupić, S. Anić, G. Schmitz, The pseudo-steady states in the model of the Bray-Liebhafsky oscillatory reaction, J. Chem. Soc. Faraday Trans. Vol. 93 (1997) 21472152.

[21] Lj. Kolar-Anić, Ž. Čupić, G. Schmitz, S. Anić, Improvement of the stoichiometric network analysis for determination of instability conditions of complex nonlinear reaction systems, Chem. Eng. Sci. Vol. 65 (2010) 3718-3728.

[22] Lj. Kolar-Anić, Ž. Čupić, V. Vukojević, S. Anić, Dinamika nelinearnih procesa, Fakultet za fiziku hemiju, Univerzitet u Beogradu, Beograd, 2011. (in Serbian)

[23] S. MAĆEŠIĆ, Ž. ČUPIĆ, S. ANIĆ, Model of the nonlinear reaction system with autocatalysis and autoinhibition: Stability of dynamic states, Hemijska industrija, Vol. 66 (2012) 637-646.

[24] S. MaĆEŠIĆ, Ž. ČUPIĆ, S. AnIĆ, LJ. KolaR-Anić, Autocatalator as the source of instability in the complex non-linear neuroendocrine model, International Journal of Non-Linear Mechanics Vol. 73, (2015) 25-30. 
[25] V. Marković, Ž. Čupić, A. IVAnović, Lu. Kolar-Anić, The Stability of the Extended Model of Hypothalamic-Pituitary-Adrenal (HPA) Axis Examined by Stoichiometric Network Analysis ( SNA), Russ. J. Phys. Chem. A Vol. 85 (2011) 2327-2335.

[26] V. M. Marković, Ž. Čupić, S. Maćešść, A. Stanojević, V. Vukojević, LJ. KolAR-ANIĆ, Modeling cholesterol effects on the dynamics of the hypothalamicpituitary-adrenal (HPA) axis, Mathematical Medicine and Biology (2014) in press, doi:10.1093/imammb/dqu020.

[27] G. Nicolis, I. Prigogine, Self-organization in Non-equilibrium Systems, John Wiley and Sons, Inc, New York, NY, 1977.

[28] G. Nicolis, I. Prigogine, Exploring Complexity, Freeman, New York, NY, 1989.

[29] G. Nicolis, Introductions to Nonlinear Science, Cambridge University Press, Cambridge, 1995.

[30] G. M. Panchenkov, V. P. Lebedev, Chemical Kinetics and Catalysis, MiR, Moscow, 1976.

[31] I. Prigogine, Time, structure and fluctuations, Nobel Lecture in Chemistry, 1977.

[32] J. Ross, I. Schreiber, M. O. Vlad, Determination of Complex Reaction Mechanisms Analysis of Chemical, Biological, and Genetic Networks, Oxford University Press, Oxford, 2006.

[33] G. Schmitz, Cinetique de la reaction de Bray, J. Chim. Phys. Vol. 84 (1987) 957-965.

[34] G. Schmitz, Etude du Braylator par la methode de Clarke, J. Chim. Phys. Vol. 88 (1991) 15-25.

[35] G. Schmitz, Lu. Kolar-Anić, S. Anić, Ž. ČUPić, Stoichiometric Network Analysis and Associated Dimensionless Kinetic Equations. Application to a Model of the Bray-Liebhafsky Reaction, J. Phys. Chem. A, Vol. 112 (2008) 13452-13457.

[36] S. K. ScotT, Chemical Chaos, Clarendon Press, Oxford, 1991.

[37] S. Veluković, Hemijska Kinetika, Graðevinska Knjiga, Beograd, 1969.

[38] A. M. ZнавотіnskiI, Kontsentratsionniye avtokolebaniya, Nauka, Moscow, 1974. 